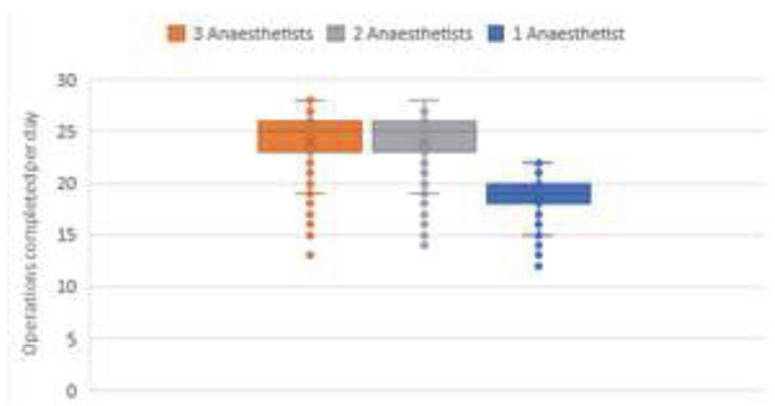

Abstract 85 Figure 3 Block room staffing effects

Results The number of completed operations were compared (figure 2) The standard configuration completed 19 operations over three operating theatres (SD 0.99), in comparison the addition of a block room increased this to 23.8 (SD 2.66). The modelling of staffing effects (figure 3) showed that throughput could be sustained despite a reduction in anaesthetic staff.

Conclusions We used simulation modelling to test the possible benefits of implementing a block room, as well as the likely impact of changes to staffing on its patient throughput. This offers useful insights into the planning and organisation of a new pathway before committing to the cost and disruption associated with service reconfiguration.

\section{SERRATUS ANTERIOR PLANE BLOCK (SAPB) IN SEVERE CHEST TRAUMA WITH MULTIPLE RIB FRACTURES (MRF): OPTIMAL COMBINATION BETWEEN ANALGESIA AND IMPROVED LUNG FUNCTION?}

${ }^{1} \mathrm{M}$ Mazzocchi ${ }^{*},{ }^{2} \mathrm{~B}$ Mascia, ${ }^{2} \mathrm{~S}$ Bonaiti, ${ }^{2} \mathrm{~A}$ Pellegrini, ${ }^{2} \mathrm{~A}$ Colombo, ${ }^{2} \mathrm{~A}$ Stella, ${ }^{3} \mathrm{~F}$ Torresani, ${ }^{2} \mathrm{G}$ Bruschi, ${ }^{2} \mathrm{~F}$ Mojoli. ${ }^{1}$ Galeazzi Orthopaedic Institute, I.R.C.C.S. (Scientific Institute for Research, Hospitalization and Health Care), Milan, Italy; ${ }^{2}$ Foundation Polyclinic San Matteo, I.R.C.C.S. (Scientific Institute for Research, Hospitalization and Health Care), Pavia, Italy; ${ }^{3}$ Sant'Anna Hospital, Como, Italy

10.1136/rapm-2021-ESRA.86

Background and Aims 10\% of polytraumatized patients have multiple rib fractures. Associated severe pain can lead to hypoventilation and respiratory complications in $31 \%$ of cases $^{1}$. 'Opioid-sparing analgesia' as epidural and multimodal analgesia are recommended ${ }^{2}$. We hypotize that $\mathrm{SAPB}^{3,4}$ can provide effective, safe and long-lasting analgesia (thanks to dexamethasone added to local anaesthetic) and reduce respiratory complications, enhancing recovery of a better mechanical lung function.

Methods 15 patients(age 64 \pm 15 ) hospitalized for polytrauma with $\operatorname{MRF}(8 \pm 4)$ and severe pain(NRS $>4)$, impairing maximal inspiration and coughing, were treated with multimodal analgesia + SAPB(ethical committee approval obtained). We injected levobupivacaine $0.25 \% 30 \mathrm{ml}$ and dexamethasone (8 $\mathrm{mg}$ ) within the myofascial plane of serratus anterior. We registered respiratory rate(RR), heart rate(HR) and NRS at rest (NRSrest), during maximal inspiration(NRSdeep) and coughing (NRScough) before SAPB(T0), after 15 minutes(T1) and after 4 hours(T2). At T0 and T2, lung ultrasound(LUS) and diaphragmatic ultrasound(DUS) were performed. Incidence of opioid-related side effects and respiratory complications were recorded.

Results We observed a significant reduction in NRSrest, NRSdeep, NRScough, HR and RR at both T1 and T2 in all patients. LUS revealed a significant improvement in lung aeration, DUS showed a significant increase in diaphragm excursion. Duration of analgesia was greater than 30 hours in most patients, greater than 48 hours in almost 50\%. No patient asked for rescue-analgesia. No patient had respiratory complications.

Conclusions Both clinical and instrumental data suggest that SAPB is safe and provides effective opioid-sparing analgesia. Pain reduction allows improvement in pulmonary aeration and diaphragmatic function, suggesting a possible role in prevention of pulmonary complications.

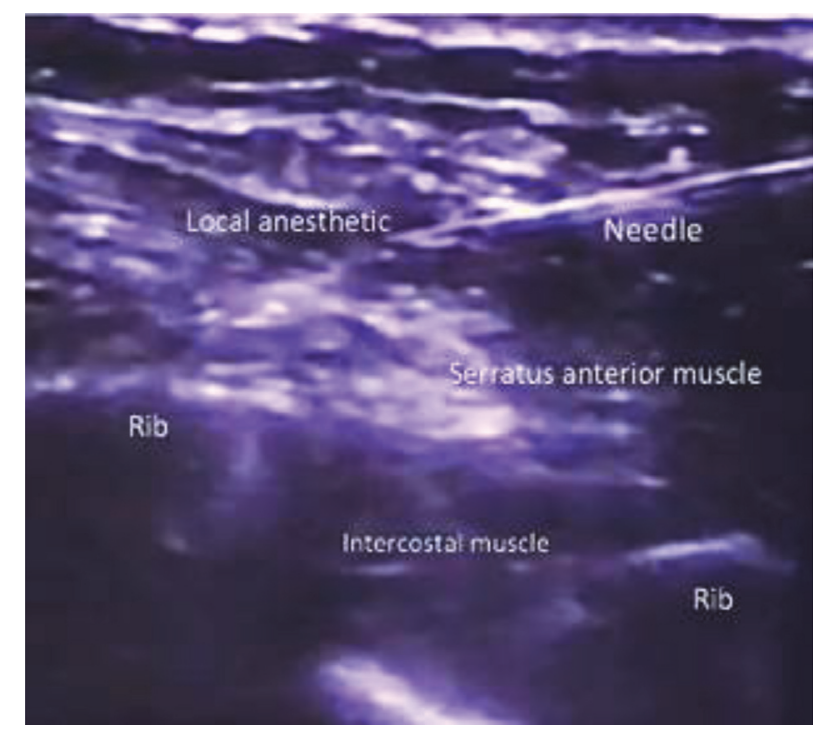

Abstract 86 Figure 1

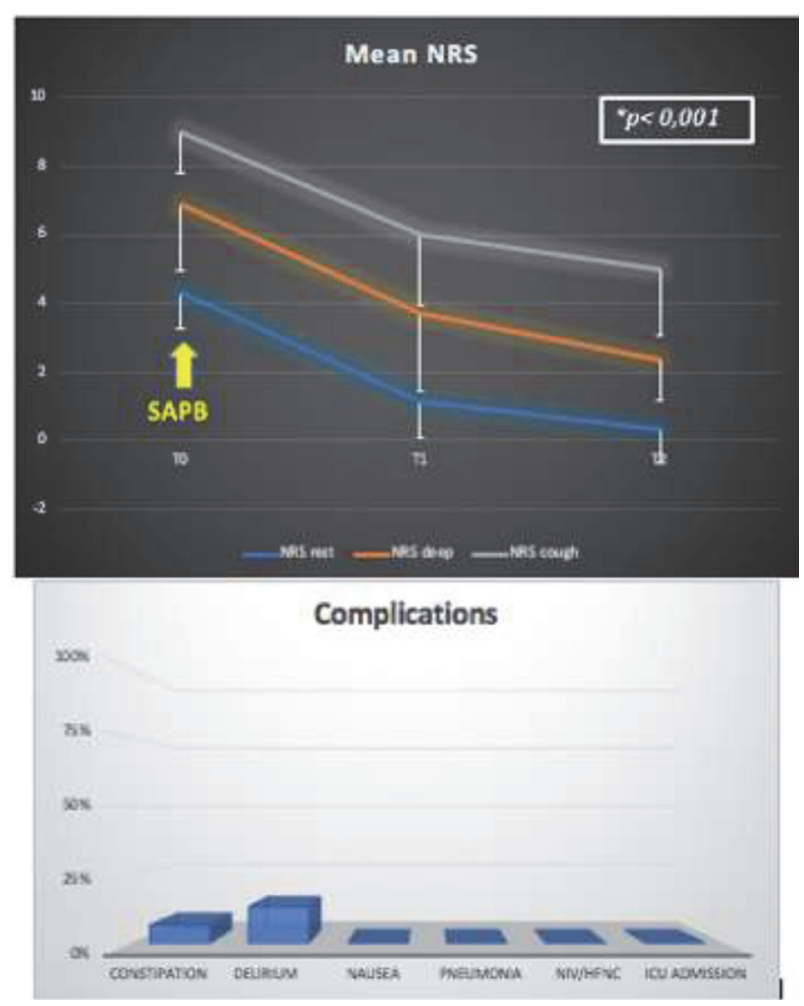

Abstract 86 Figure 2 

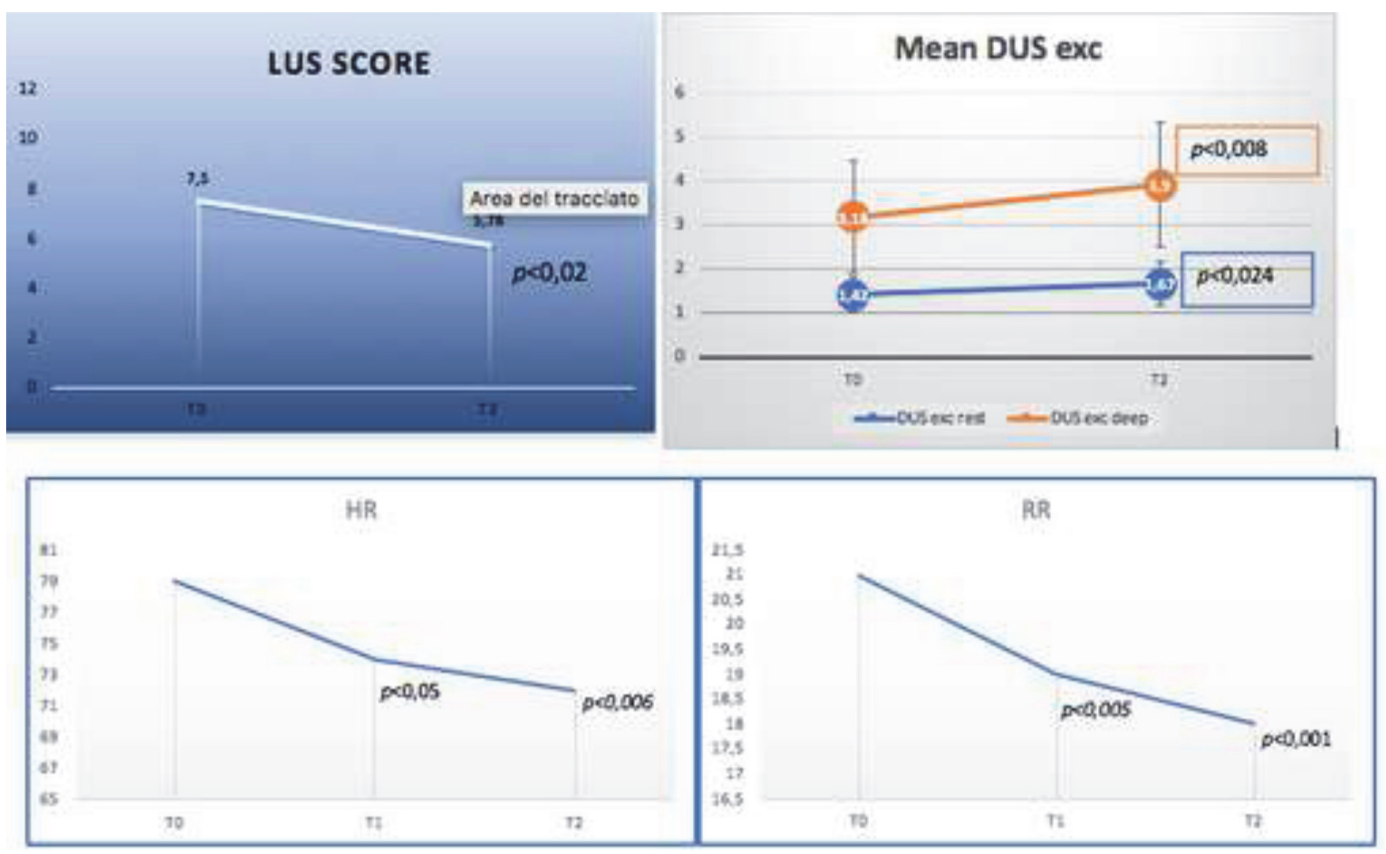

Abstract 86 Figure 3

\section{THE ERECTOR SPINAE PLANE BLOCK (ESPB) IN INTERMITTENT DOSES FOR VIDEO ASSISTED THORACOSCOPIC SURGERY (VATS)}

F Longo*, E Tomaselli, M Martuscelli, A Riccetti. Campus Bio-Medico University Hospital, Rome, Italy

\subsection{6/rapm-2021-ESRA.87}

Background and Aims VATS has become the first treatment for patients undergoing lobectomy. Recent reports showed that ESPB improved postoperative analgesia after VATS.

ESPB is a technique introduced by Forero et al. Local anesthetic is injected between the erector spinae muscle and transverse process. Recent studies regarding the use of boluses for an ESPB catheter suggested that some patients do better with mandatory boluses than continuous infusions.

We report the use of the ESPB in intermittent boluses in a 69 yrs man, with important comorbidities that underwent upper right pulmonary VATS lobectomy.

Methods After induction of general anesthesia, the patient was placed in lateral position and a linear-array transducer was placed in longitudinal orientation. After local anesthesia, an ESPB at T5 level is performed using an 18G Thuoy needle. After a bolus of $20 \mathrm{ml}$ of ropivacaine $0.5 \%$, a perineural catheter was placed, $6 \mathrm{~cm}$ beyond the needle tip (figure 1). After the surgery, a bolus of ropivacaine $0,2 \%(10 \mathrm{ml})$ was administered and subsequently boluses of $20 \mathrm{ml}$ of ropivacaine $0,2 \%$ were administered every 8 hours up to 48 hours after surgery. Results NRS pain score decreased immediately after every bolus. The patient performed successful respiratory rehabilitation. The length of hospital stay was reduced, and the patient reported comfort and satisfaction.

Conclusions The ESPB in intermittent boluses had no side effects and allowed a reduction in the consumption of opioids and therefore of post-operative nausea and vomiting. It could be an alternative to epidural anesthesia or Serratus Anterior Plane Block.

\section{COVID AND THE BRI: A HIP FRACTURE STORY}

${ }^{1,2}{ }^{2}$ Hennebry*, ${ }^{1,2} \mathrm{E}$ Court, ${ }^{1} \mathrm{~A}$ Bartlett, ${ }^{1} \mathrm{~S}$ Dennis. ${ }^{1}$ University Hospitals Bristol, Bristol, UK; ${ }^{2}$ University of Bristol, Bristol, UK

\subsection{6/rapm-2021-ESRA.88}

Background and Aims The COVID-19 pandemic has had huge implications on healthcare; we aimed to quantify the impact hip fracture provision at the Bristol Royal Infirmary.

Methods We undertook an interrupted time series analysis across four months, representing pre-COVID-19, the first wave, the second wave, and now. We compared the following factors: number of \#NOF cases, delays to theatre, delays due to lack of theatre capacity, and length of stay. We also looked at interruptions to patient care, including ward changes and the closure of Weston General.

Results With patients in lockdown, number of \#NOF were expected to reduce; there was no evidence for this.

During the first wave, the average length of stay was 11.1 days, compared to 26.0 days pre-COVID-19 (p-value 0.0016). Now, though lengths of stay are gradually increasing, they are still significantly shorter than pre-COVID-19, with an average of 17.1 days (p-value 0.04 ). The number of patient delays did not significantly change.

Additionally, Weston General closing increased both case numbers and delays to theatre, with cases rising from 14 to $26(+85.7 \%)$ and delays to theatre from 5 to 17 $(+240 \%)$.

Conclusions Patient stays are still shorter than pre-pandemic; whether this is a valuable lasting change will be evident in the coming months. Weston General becoming COVID-only put strains on the capacity of the BRI, demonstrated by the increased caseload and resulting theatre delays.

Several patients were significantly delayed without a medical reason: some for up to a week, despite being listed repeatedly. Prioritisation of theatre lists needs to be reviewed. 